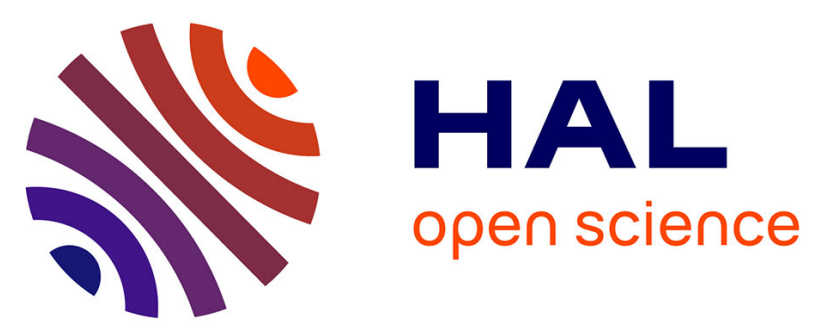

\title{
Homothetic Method to Compute Winding Losses in the Design of Power Inductors
}

\author{
Andre Furlan, Alvaro Morentin, Guillaume Fontes, Guillaume Delamare, \\ Marcelo Heldwein, Thierry Meynard
}

\section{- To cite this version:}

Andre Furlan, Alvaro Morentin, Guillaume Fontes, Guillaume Delamare, Marcelo Heldwein, et al.. Homothetic Method to Compute Winding Losses in the Design of Power Inductors. 2019 IEEE 15th Brazilian Power Electronics Conference and 5th IEEE Southern Power Electronics Conference (COBEP/SPEC), Dec 2019, Santos, Brazil. pp.1-6, 10.1109/COBEP/SPEC44138.2019.9065497 . hal-02999721

\section{HAL Id: hal-02999721 \\ https://hal.science/hal-02999721}

Submitted on 2 Dec 2020

HAL is a multi-disciplinary open access archive for the deposit and dissemination of scientific research documents, whether they are published or not. The documents may come from teaching and research institutions in France or abroad, or from public or private research centers.
L'archive ouverte pluridisciplinaire HAL, est destinée au dépôt et à la diffusion de documents scientifiques de niveau recherche, publiés ou non, émanant des établissements d'enseignement et de recherche français ou étrangers, des laboratoires publics ou privés. 


\title{
Homothetic Method to Compute Winding Losses in the Design of Power Inductors
}

\author{
Andre Furlan' ${ }^{1,2}$, Alvaro Morentin ${ }^{1}$, Guillaume Fontes ${ }^{1}$, Guillaume Delamare ${ }^{1}$, Marcelo L. Heldwein ${ }^{2}$, \\ Thierry Meynard ${ }^{1,3}$ \\ 1 Power Design Technologies, Toulouse, France \\ 2 Power Electronics Institute (INEP), Federal University of Santa Catarina (UFSC), Florianopolis, Brazil' \\ ${ }^{3}$ LAPLACE, Universite de Toulouse, CNRS, INPT, UPS, France'
}

\begin{abstract}
This work proposes an improvement for the consideration of ac winding losses in the design process of inductors to be employed in power converters, where the geometrical parameters of the inductors are not known before starting the design. The presented method consists of the pre-computation of a homothetic multivariate regular grid surface by finite element simulations, from which the winding resistance can be obtained by direct multivariate linear interpolation. Special attention is given to Dowell's analytical formulations. These are often used in the design but typically with little details given on how the final expressions are derived. The results with the new method are shown to be more accurate than the analytical calculations over a wide range of design points and, thus, are a useful design tool.

Index Terms-Winding resistance, finite element analysis, magnetic components, air gap
\end{abstract}

\section{INTRODUCTION}

Dowell's equations [1] are often used to calculate the equivalent ac resistance $\left(R_{A C}\right)$ of inductor windings, taking skin and proximity effects into account. These equations are derived through a $1 \mathrm{D}$ approximation to calculate the window magnetic field under certain simplifying hypotheses. However, inductors are generally gapped, and this 1D approximation is not applicable since the fringing field of the air gap cannot be described in a 1D manner [2]. Gapped inductors can be accurately analyzed using the finite element method (FEM) with the computation efforts and CPU time that this represents. When a fast computation of the $R_{A C}$ is needed, to avoid a computationally intensive finite element simulation, some works applied tuned equations based in Dowell's methods [3] [4]. In such works, additional coefficients are introduced through a data fitting process against FEM results. The derived relation coefficients depend on the inductor geometrical parameters. Consequently, this approach can be used only for specific geometries. Some analytical methods for gapped inductors are presented in [2] [5] [6]. Nevertheless, a final validation with a FEM simulation is indispensable.Fig. 1 describes a typical power inductor design procedure.

After a fast design, using the area product approach $(A w A c)$ [7], with the geometric parameters, the winding loss can be evaluated through different methods. Following this evaluation, the calculated and projected power losses are compared.

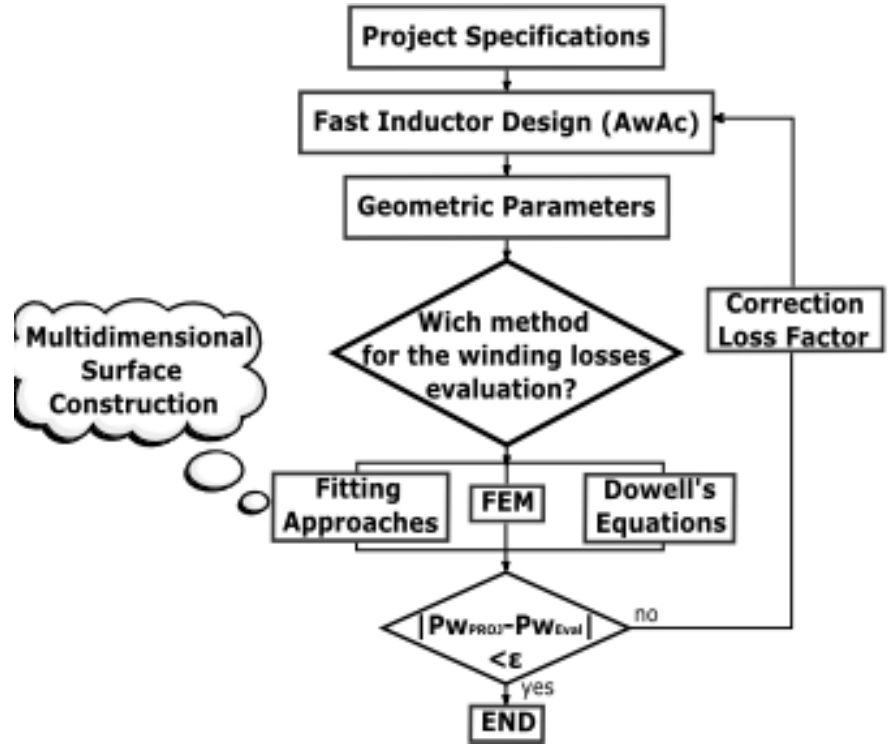

Fig. 1. Inductor design procedure. 
If the error is greater than the tolerance, the design process is repeated once again, using a correction loss factor.

In this work, a general approach based on the interpolation of a surface that is pre-computed using 2D FEM is proposed to obtain the $R_{A C}$ of a generic E-core inductor in a reduced CPU time. Thus, generating a process that is adequate for use in iterative and optimization design tools. The homothetic properties of these structures as well as relations between different conductivities are explored to reduce the number of variables. Generating this surface requires a high computational effort. Nevertheless, it can be used in the design of an arbitrary number of inductors after being computed offline.

First, the topology of the studied inductor is presented, and Dowell's equations are derived with two different approaches: neglecting the gap in the calculation of the orthogonal magnetic field $\left(H_{E}\right)$ and considering the gap in the evaluation of $H_{E}$.

After describing the formulation, the homothetic and conductivity relations are studied to reduce the number of variables of the problem. Different values of inductor geometric parameters are combined, and many FEM simulations have been run to establish a reduced multidimensional FEM surface. $R_{A C}$ is then evaluated through direct multivariate linear interpolation from the FEM results. The resulting values are shown to match FEM simulations more closely than analytical formulations.

\section{CHARACTERISTICS OF THE FOIL INDUCTOR}

Fig. 2 describes the cross section of the right-side corewinding window of an E-core inductor that is built with foil conductors using air gaps in the center and outer legs.

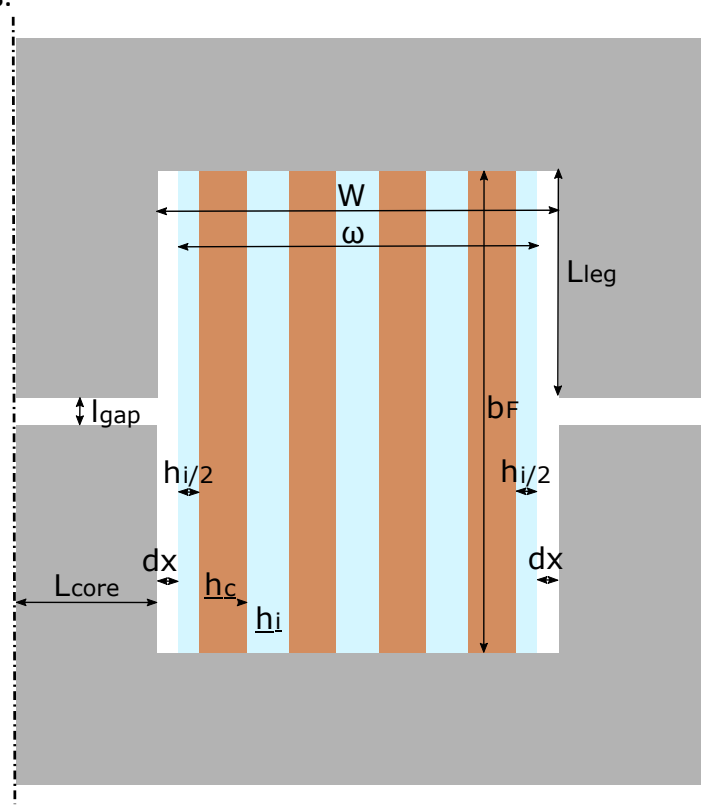

Fig. 2. Cross section of the right-side core-winding window of an E-core inductor.

The following parameters are defined:

- $f$ : excitation frequency $[\mathrm{Hz}]$

- $N$ : number of turns;

- Lcore: core leg width [m];

- $W$ : core window width [m];

- $w$ : winding window width $[\mathrm{m}]$;

- Lleg: core leg height [m];

- $b_{F}$ : core window total height $[\mathrm{m}]$;

- $h_{c}$ : foil conductor thickness [m];

- $h_{i}$ : insulation thickness [m];

- $I_{\text {gap }}$ : air gap length [m];

- $d x$ : distance between coil insulation and vertical core legs $(d x=(W-w) / 2)[\mathrm{m}]$

- $\sigma$ : foil conductivity $[\mathrm{S} / \mathrm{m}]$;

- $\mu_{c r}$ : core relative permeability; 
- $k_{w}$ : winding fill factor. Ratio between the winding section area and the winding window area without spacing $\left(k_{w}=N\right.$. $\left.h_{c} / w\right)$.

\section{Classical analytical Formulation}

Two different approaches for Dowell's equations are analyzed in this section. The first approach is widely used in previous works: it was developed for ungapped cores, resulting in the classical Dowell's equation. The second approach considers the gap in the expression of the orthogonal field $\left(H_{E}\right)$. Usually, little details are given about the formulation and its application.

Classical methods to compute $R_{A C}$ are based in the orthogonality existing between skin effect and proximity effect in conductors. This makes possible to decouple the two effects and simplifies the analysis [8]. In the next subsections, these effects will be described in terms of losses for a sinusoidal excitation at frequency $f$ in order to calculate $R_{A C}$.

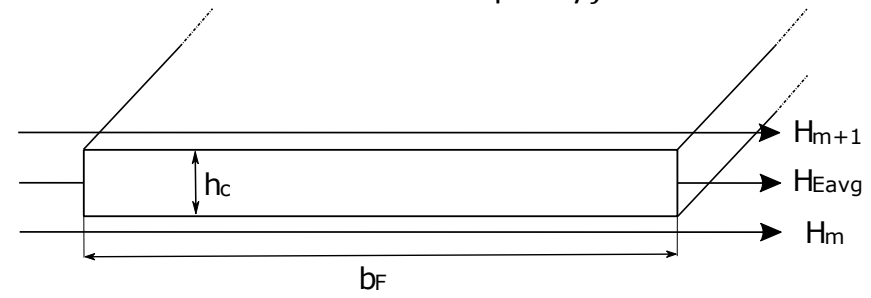

Fig. 3. Cross section of a foil conductor that is influenced by an external magnetic field $\left(H_{E}\right)$

\section{A. Skin Effect}

The skin-effect losses for a single winding layer (including DC losses) per unit length is calculated as [2]

$$
P_{S k i n}=F_{S k i n} \cdot r_{D C} \cdot \widehat{I}^{2}
$$

where $I \mathrm{~b}$ is the peak current flowing through the coil, $\Delta$ is the foil conductor thickness normalized to the skin depth $(\sqrt{ }$ $\left.h_{c} / \delta\right), \delta$

is the skin depth $\left(1 / \pi \mu_{0} \sigma f\right), \mu_{0}$ is the vacuum permeability, $r_{D C}$ is the single layer $D C$ resistance $\left(1 /\left(\sigma b_{F} h_{C}\right)\right)$, and $F_{\text {Skin }}$ is the factor that describes the rise of the conductor resistance due to the skin effect (2).

$$
F_{\text {Skin }}=\frac{\Delta}{4} \frac{\sinh \Delta+\sin \Delta}{\cosh \Delta-\cos \Delta}
$$

\section{B. Proximity Effect}

The losses due to the proximity effect for a single winding layer are described as [2]

PProx $=F$ Prox $\cdot r D C \cdot H_{E a v g}{ }^{2}(3)$ where $F_{\text {Prox }}$ is a factor that describes the amount of winding losses due to the proximity effect

$$
F_{\text {Prox }}=b_{F}^{2} \Delta \frac{\sinh \Delta-\sin \Delta}{\cosh \Delta+\cos \Delta}
$$

and $H_{\text {Eavg }}$ is the average value of the two magnetic fields of the two sides of the coil, illustrated in Fig. 3 and described by the following equation

$$
H_{\text {Eavg }}=\frac{H_{m}+H_{m-1}}{2}
$$

where $m$ is the number of the layer, and

$$
H_{m}=\frac{m \widehat{I}-h_{c g} l_{g}}{b_{F}}
$$

where $h_{c g}$ is the magnetic field at the center gap. Usually, this variable is not included in the Dowell's method demonstration, which would lead to a better understanding of Dowell's equations.

With (5) and (6), $H_{\text {Eavg }^{2}}$ becomes 


$$
H_{\text {Eavg }}^{2}=\left(\frac{(2 m-1) \widehat{I}}{2 b_{F}}\right)^{2}-\frac{(2 m-1) \widehat{I} h_{c g} l_{g}}{b_{F}}+\left(\frac{h_{c g} l_{g}}{b_{F}}\right)^{2}
$$

C. Total Losses

The total proximity losses per unit length for $N$ turns is

N

PProxTot $=$ FProx $\cdot r D C \cdot \mathrm{X} \mathrm{HEavg2}$

$m=1$

where

$$
\begin{aligned}
& \sum_{m=1}^{N} H_{\text {Eavg }}^{2}= \\
& \sum_{m=1}^{N}\left[\left(\frac{(2 m-1) \widehat{I}}{2 b_{F}}\right)^{2}-\frac{(2 m-1) \widehat{I} h_{c g} l_{g}}{b_{F}}+\left(\frac{h_{c g} l_{g}}{b_{F}}\right)^{2}\right] \\
& =\frac{\left(4 N^{3}-N\right) \widehat{I}^{2}}{12 b_{F}^{2}}-\frac{h_{c g} l_{g} N^{2} \widehat{I}}{b_{F}^{2}}+\frac{h_{c g}^{2} l_{g}^{2} N}{b_{F}^{2}}
\end{aligned}
$$

For an ungapped core $l_{g}=0$. Thus,

$$
\sum_{m=1}^{N} H_{\text {Eavg }}^{2}=\frac{\left(4 N^{3}-N\right) \widehat{I}^{2}}{12 b_{F}^{2}}
$$

and

$$
\sum_{m=1}^{N} H_{E a v g}^{2}=\frac{\left(N^{3}-N\right) \widehat{I}^{2}}{12 b_{F}^{2}}
$$

$$
h_{c g}=N \widehat{I} / 2 l_{g} \text {. }
$$

for

The total losses per unit length including proximity and skin effects can be found with

$$
\begin{gathered}
N \\
P \text { Tot } \\
m=1 \\
m=1
\end{gathered}
$$

1) Ungapped Core: For ungapped cores (12) becomes

$$
\begin{aligned}
& P_{\text {Tot }}=R_{D C} \widehat{I}^{2} \frac{\Delta}{4}\left(\frac{\sinh \Delta+\sin \Delta}{\cosh \Delta-\cos \Delta}+\right. \\
&\left.\frac{4 N^{2}-1}{3} \frac{\sinh \Delta-\sin \Delta}{\cosh \Delta+\cos \Delta}\right)
\end{aligned}
$$

where $R_{D C}=N r_{D C}$. Thus, it follows that,

$$
\begin{aligned}
& R_{A C}=R_{D C} \frac{\Delta}{2}\left(\frac{\sinh \Delta+\sin \Delta}{\cosh \Delta-\cos \Delta}+\right. \\
&\left.\frac{4 N^{2}-1}{3} \frac{\sinh \Delta-\sin \Delta}{\cosh \Delta+\cos \Delta}\right)
\end{aligned}
$$

Equation (14) presented in [2] is equivalent to classic Dowell's formulation presented in several works, in particular [9]. Indeed, by applying (15) into (14), the classic Dowell's equation (16) is obtained. 


$$
\begin{aligned}
& \frac{\sinh \Delta+\sin \Delta}{\cosh \Delta-\cos \Delta}= \\
& 2 \frac{\sinh 2 \Delta+\sin 2 \Delta}{\cosh 2 \Delta-\cos 2 \Delta}-\frac{\sinh \Delta-\sin \Delta}{\cosh \Delta+\cos \Delta} \\
& R_{A C}=R_{D C} \Delta\left(\frac{\sinh 2 \Delta+\sin 2 \Delta}{\cosh 2 \Delta-\cos 2 \Delta}+\right. \\
& \left.\frac{2\left(N^{2}-1\right)}{3} \frac{\sinh \Delta-\sin \Delta}{\cosh \Delta+\cos \Delta}\right)
\end{aligned}
$$

2) Gapped Core: For the gapped core shown in Fig. 2, (12) becomes

$$
\begin{aligned}
& P_{\text {Tot }}=R_{D C} \widehat{I}^{2} \frac{\Delta}{4}\left(\frac{\sinh \Delta+\sin \Delta}{\cosh \Delta-\cos \Delta}+\right. \\
&\left.\frac{N^{2}-1}{3} \frac{\sinh \Delta-\sin \Delta}{\cosh \Delta+\cos \Delta}\right)
\end{aligned}
$$

the above expression gives,

$$
\begin{aligned}
& R_{A C}=R_{D C} \frac{\Delta}{2}\left(\frac{\sinh \Delta+\sin \Delta}{\cosh \Delta-\cos \Delta}+\right. \\
&\left.\frac{N^{2}-1}{3} \frac{\sinh \Delta-\sin \Delta}{\cosh \Delta+\cos \Delta}\right)
\end{aligned}
$$

Equation (18) differs from the classical approach (16), and it is found in the literature much less frequently, in particular (18) is found in [3].

\section{Multi-dimensional SURFACE DESIGN}

The construction of a regular homothetic multi-dimensional surface is proposed in this study. This surface can be used in the Fitting Approaches described in Fig. 1, allowing a simple multivariate interpolation to evaluate $R_{A C}$ for generic gapped E-core inductors. In the multivariate problem, linear multidimensional interpolation is an attractive choice due to its computation speed and simplicity.

Throughout this section, the homothetic properties of the proposed surface, as well as relations between different conductivities, are explored to reduce the number of variables. Finally, the surface construction is described.

\section{A. Homothetic}

A possible approach to reduce the number of variables is to consider fixed shapes of magnetic cores with dimensions that vary according to a homothetic law [10].

According to Dowell's equations, for two inductors with foil conductor thicknesses $h_{c 1}$ and $h_{c 2}$, to have the same $R_{A C} / R_{D C}$, we need to have $\Delta_{1}=\Delta_{2}$, i.e. $h_{c 1} / \delta_{1}=h_{c 2} / \delta_{2}$. For homothetic inductors, $N_{1}=N_{2}$ and $k_{w 1}=k_{w 2}$, thus,

$$
w_{1} / \delta_{1}=w_{2} / \delta_{2}
$$

Therefore, instead of working directly with the frequency in our design of experiments, we can use the foil conductor thickness normalized to the skin depth $(w / \delta)$. The geometric compensation is contained inside this relation. For the same foil materials $\left(\sigma_{1}=\sigma_{2}\right)$ applying the relation (19) and the skin depth equation leads to

$$
f_{2}=f_{1} \cdot K_{g 12}^{2}
$$

where $K_{g 12}=w_{1} / w_{2}$ is the geometric factor of two homothetic inductors.

All geometric variables must follow the geometric factor to take advantage of the homothetic property, except the $w / \delta$ ratio, where the geometric factor is already implicit. To have a homothetic surface, we can set a geometric parameter and change the other parameters in function of their relations with it. 


\section{B. Conductivity Relations}

Another important advantage of working with the $w / \delta$ ratio is that the same surface, constructed by fixing a winding material, can be used for different coil conductivities. The conductive factor is also implicit in the thickness of the foil conductor normalized to the skin depth. For two identical inductors with only different winding material, the relation (19) becomes:

$$
\delta 2=\delta 1=\Rightarrow f_{2}=f_{1} K \sigma 12
$$

where $K_{\sigma 12}=\sigma_{1} / \sigma_{2}$ is the conductivity factor of two identical inductors with different foil conductivities, $\sigma_{1}$ and $\sigma_{2}$, respectively.

\section{Surface Construction}

The multivariate regular grid surface implies that all possible combinations between the different variables are covered. To generate this surface, the value of the fixed parameter needs to be chosen. Upper and lower limits for the remaining variables are defined. The discretization of the resulting intervals and a FEM simulation for each of the possible combinations are done in the sequence. The resulting total number of points on the surface depends on the number of different discrete values of each variable.

The flowchart illustrated in Fig. 4 describes the construction of the proposed homothetic surface. Subsequently, each item of this flowchart will be discussed.

1) Variables of the Problem : In order to construct a regular grid surface, the relative permeability of the core is fixed to 2100, and aluminum foil conductors are used for the inductor winding. Eight variables result, namely: $f, N, W, w, L$ core,

Lleg, lgap, and $k w$.

2) Fixed-value Parameter: The variable $W$ was chosen to be fixed. Then the following function represents the multidimensional surface

$$
R_{A C} / R_{D C}=F_{s}\left(N, k_{w}, \frac{w}{\delta}, \frac{w}{W}, \frac{L_{\text {core }}}{W}, \frac{L_{\text {leg }}}{W}, \frac{l_{\text {gap }}}{W}\right)
$$

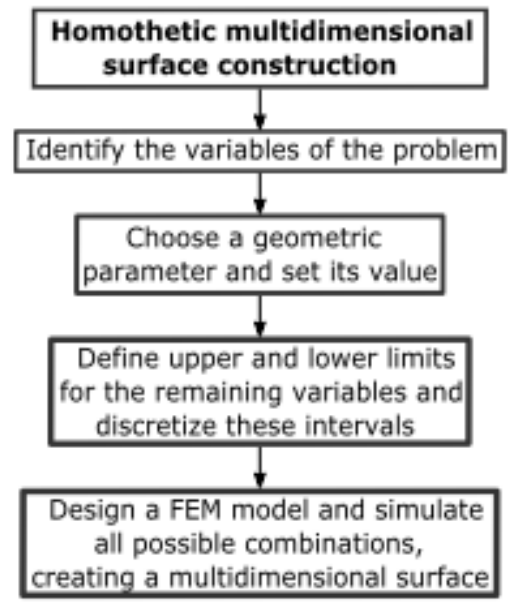

Fig. 4. Proposed Homothetic Surface.

It can be noted that all variables of (22) are dimensionless, which is a typical characteristic of a homothetic problem.

In terms of simulation, $W$ was fixed to a small value $(0.05 \mathrm{~m})$. Although the surface is constructed with a fixed $W$, due to the homothetic property, as we are working with geometric normalized variables, this surface can be used to interpolate any value of $W$. A reduced maximum frequency for larger topologies is identified by considering (20), which is not a problem for the design since higher frequencies tend to reduce magnetics size.

3) Discretization of Parameters: The next step is to determine the range of the ratios between the remaining geometric variables and the fixed-value parameter $\left(w / W, L_{\text {core }} / W, L_{\text {leg }} / W\right.$ and $\left.l_{\text {gap }} / W\right)$. To define upper and lower limits for the core variables $L_{\text {core }} / W$ and $L_{\mathrm{leg}} / W$, the $\mathrm{E}$ cores present in [12] were taken as reference. The minimum and the maximum air gap normalized by the core window select for our test surface were $W / 200$, and $W / 2$, respectively. The winding window ( $w$ ) was set between $50 \%$ and $99.8 \%$ of $W$. Finally, the winding fill factor was set between 0.2 and 0.8 , and the number of turns was 
limited to 10 , to reduce the calculation time to construct the surface test. The discretization of the variables intervals is described below:

$$
\begin{aligned}
& {[N]=[1,5,10]} \\
& {\left[L_{\text {core }} / W\right]=[0.4,0.8,1.2]} \\
& {[w / W]=[0.5,0.7,0.8,0.98,0.99,0.998]} \\
& \quad\left[L_{\text {leg }} / W\right]=[1.0,1.5,2.0,2.5,3.0] \\
& {\left[\text { lgap }_{\text {gap }} / W\right]=[0.005,0.05,0.5]} \\
& {\left[k_{w}\right]=[0.2,0.4,0.6,0.8]} \\
& {[w / \delta]=[0.1,10,25,100,250,500]}
\end{aligned}
$$

The total number of FEM simulations to construct a regular grid surface with the above variables intervals is: $\operatorname{range}(N)$. $\operatorname{range}\left(\frac{L_{\text {care }}}{W}\right) \cdot \operatorname{range}\left(\frac{w}{W}\right) \cdot \operatorname{range}\left(\frac{\text { leg }}{W}\right) \cdot \operatorname{range}\left(\frac{\text { gap }}{W}\right) \cdot$ $\operatorname{range}\left(k_{w}\right) \cdot \operatorname{range}\left(\frac{w}{\delta}\right)=19440$.

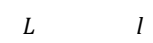

4) FEM Design: The 2D FEM simulations have been carried out with the FEMM software [13]. The harmonic magnetodynamic formulation is used to calculate the equivalent

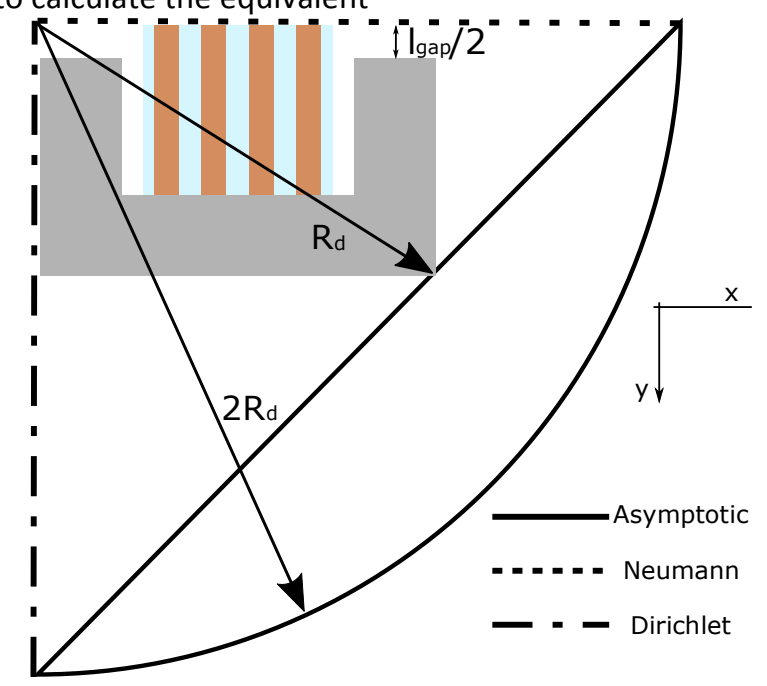

Fig. 5. Reduced FEM Domain

resistance of the windings. Details of its formulation are found in [14].

Due to the symmetry of the problem, only one-quarter of the domain is modeled as shown in Fig. 5. The application of Asymptotic Boundary Conditions ( $A B C$ ) is proposed for the FEMM model, which is a way to approximate an open boundary, other than truncation, as described in [15]. Classical Neumann and Dirichlet boundary conditions are applied for the symmetric axes of the model, $x$ and $y$, respectively.

Mesh refinement is essential to increase the accuracy of finite element calculations. The mesh refinement must be compatible with the skin depth $(\delta)$ of the winding. Usually, 1.5 to 2 elements per skin depth are required for a correct solution [14]. In this work, the conductors were divided into layers of $\delta$-thickness from the surface layer up to a depth of $3 \delta$. Assuming the maximum size of each triangular element is equal to $\delta / 2$ in these layers, and outside of these layers the finite element mesh automatically generated by FEMM, as shown in Fig. 6 , a sufficient level of refinement is achieved leading to consistent results. 


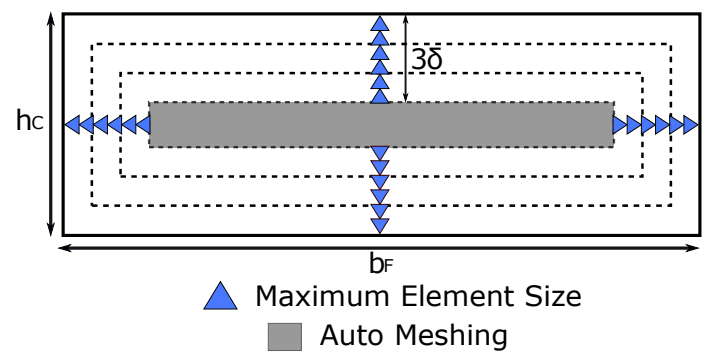

Fig. 6. Mesh Refinement Criteria

\section{RESUltS}

In order to test the accuracy of the new method, the $R_{A C} / R_{D C}$ values of 1000 randomly-chosen design points are obtained from the constructed surface by direct multivariate linear interpolation.

The interpolation is implemented using the SciPy library, an open source scientific library for Python used for scientific and technical computing [11]. Particularly, the interpolation is done with the scipy.interpolate.RegularGridlnterpolator class, an interpolation method on a regular grid in arbitrary dimensions. A linear interpolation method has been chosen and will be called to interpolate each required design.

The random points are created between the upper and lower limits of the variables of the problem described in (23). For the width of the core window $(W)$, fixed in our FEM simulation, we explore the homothetic property with random values between 0.005 and $1 \mathrm{~m}$, spanning a very wide range of applications. The random variables are described in (24).

$$
\begin{aligned}
& N_{\text {Rand }}=\operatorname{int}(\operatorname{random}(1,10)) L_{\text {core }} / W_{\text {Rand }}=\operatorname{random}(0.4,1.2) w / W_{\text {Rand }}=\operatorname{random}(0.5,0.998) L_{l e g} / W_{\text {Rand }}= \\
& \operatorname{random}(1,3) \\
& l_{\text {gap }} / W_{\text {Rand }}=\operatorname{random}(0.005,0.5) k_{w R a n d}=\operatorname{random}(0.2,0.8) w / \delta_{\text {Rand }}=\operatorname{random}(1,500) \\
& W_{\text {Rand }}=\operatorname{random}(0.005,1)
\end{aligned}
$$

Formulations (18), (16) and the proposed interpolation method are compared in Fig. 7 relative to FEM simulations. The errors are respectively defined as

$$
E R R_{\text {ACtest } \%}=100 \times\left(\frac{R_{\text {ACtest }}-R_{A C F E M}}{R_{A C F E M}}\right)
$$

where $R_{A C t e s t}$ can be the $R_{A C}$ calculated with (16), (18) or by the interpolation method.

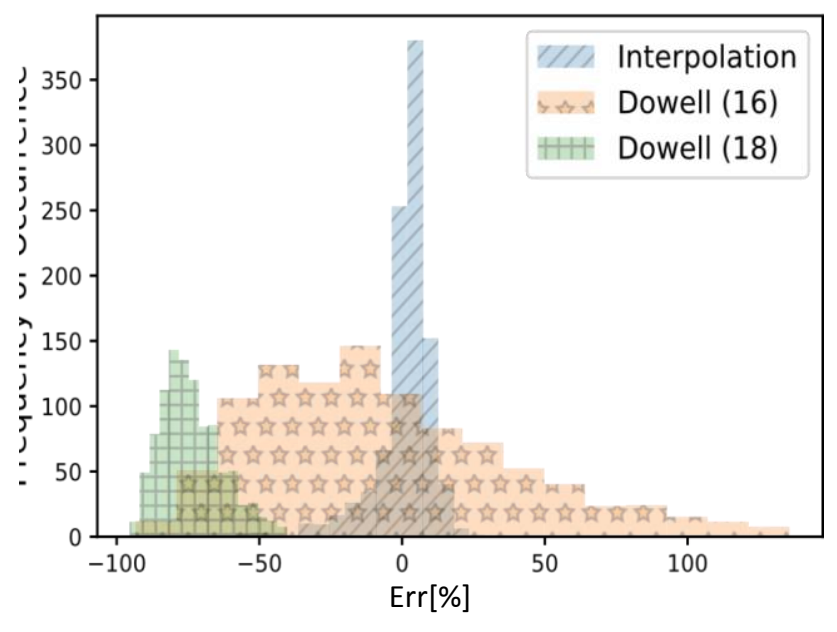

Fig. 7. $R_{A C}$ errors: Dowell (16), Dowell (18) and Interpolation.

The results with the proposed method are more accurate than analytical calculations as shown in Fig. 7 . The mean absolute error applying (16) is lower than the mean absolute error of (18). Otherwise, with (16), the data values are more spread out 
from the mean. The comparison is more evident in Tab. I, with the evaluation of the mean and the standard deviation (Std) of $\left|E R R_{A C t e s t} \%\right|$, maximum and minimum values of $E R R_{A C t e s t} \%$ and variance of $E R R_{A C t e s t} \%$.

As shown in Tab. I, the estimated absolute error (Mean \pm Std) in this application of the proposed method is $6.7 \pm 7.0$, a lower value comparing to $37.3 \pm 25.8(16)$ and $73.7 \pm 10.6$ (18). Although the estimated absolute error is lower with the Dowell classic formulation than with the air gap formulation, its variance is much higher when compared to the other approaches. What was expected by looking at the Fig. 7, where the Dowell (16) distribution is more spread out than the Dowell (18) distribution. Therefore, a correction factor would be more easily applied to (18) than to (16).

TABLE I

Mean , Standard Deviation (StD), Min and Max of ERrors And Variance

\begin{tabular}{l|cc|ccc}
\hline \hline \multirow{2}{*}{ Method } & $\left|E R R_{A C \%}\right| \mid E R R_{A C \%}$ & $E R R_{A C \%} E R R_{A C \%} E R R_{A C \%}$ \\
& Mean & Std & Min & Max & Var \\
\hline Dowell(16) & 37.3 & 25.8 & -93.3 & 135.8 & 2011 \\
\hline Dowell(18) & 73.7 & 10.6 & -95.2 & -40.1 & 113 \\
\hline Interpolation & 6.7 & 7.0 & -58.1 & 29.1 & 92 \\
\hline
\end{tabular}

\section{CONCLUSIONS}

This work proposed an improvement for taking into account ac winding losses in the inductor design process, where the geometrical parameters are not defined before starting the design.

First, special attention was paid to Dowell's formulations. Several works use the Dowell's method applying (18) or (16) to calculate inductors the equivalent resistance of the windings, but, usually, no more details are given about the equations. However, it was shown that these equations give different results. Generally, both formulations can have significant errors compared to FEM simulation.

A new homothetic method was proposed for E-core inductors, improving $R_{A C}$ calculations. The presented method consists of the construction of a homothetic multivariate regular grid FEM surface, from which $R_{A C}$ is obtained through direct multivariate linear interpolation. In the surface construction, the geometric variable $W$ (width of the core window) was set, but due to the homothetic properties, the proposed surface can be used to interpolate any value of $W$. The same surface can also be used for winding materials with different conductivities.

The results with the proposed method typically are more accurate than analytical calculations. The accuracy of the new approach could be further improved by increasing the discretization of the problem variables, at the cost of more computational effort. However, this computation can be performed offline once and used a posteriori for designing an arbitrary number of different inductors.

\section{REFERENCES}

[1] P. L. Dowell, "Effects of eddy currents in transformer windings," in Proceedings of the Institution of Electrical Engineers, vol. 113, no. 8, pp. 1387-1394, August 1966.

[2] J. Muehlethaler, Modeling and multi-objective optimization of inductive power components. Zurich, Switzerland: Eidgenossische Technische" Hochschule Zurich, 2012."

[3] F. A. Holgun, R. Asensi, R. Prieto and J. A. Cobos, "Simple analytical approach for the calculation of winding resistance in gapped magnetic components," 2014 IEEE Applied Power Electronics Conference and Exposition - APEC 2014, Fort Worth, TX, 2014, pp. 2609-2614.

[4] Xi Nan and C. R. Sullivan, "An improved calculation of proximityeffect loss in high-frequency windings of round conductors," IEEE 34th Annual Conference on Power Electronics Specialist, 2003. PESC'03., Acapulco, Mexico, 2003, pp. 853-860 vol.2.

[5] A. Van den Bossche and V. Valchev, "Eddy current losses and inductance of gapped foil inductors," IEEE 2002 28th Annual Conference of the Industrial Electronics Society. IECON 02, Sevilla, 2002, pp. 1190-1195 vol.2.

[6] P. Wallmeier, N. Frohleke and H. Grotstollen, "Improved analytical modeling of conductive losses in gapped high-frequency inductors," Conference Record of 1998 IEEE Industry Applications Conference. Thirty-Third IAS Annual Meeting (Cat. No.98CH36242), St. Louis, MO, USA, 1998, pp. 913-920 vol.2.

[7] T. Meynard, Analysis and Design of Multicell DC/DC Converters Using Vectorized Models. Hoboken, NJ: Wiley, 2015, ch. 7.

[8] J. A. Ferreira, "Improved analytical modeling of conductive losses in magnetic components," in IEEE Transactions on Power Electronics, vol. 9, no. 1, pp. 127-131, Jan. 1994.

[9] Z. Shen, Z. Li, L. Jin and H. Wang, "An AC resistance optimization method applicable for inductor and transformer windings with full layers and partial layers," 2017 IEEE Applied Power Electronics Conference and Exposition (APEC), Tampa, FL, 2017, pp. 2542-2548.

[10] F. Forest, E. Laboure, T. Meynard and M. Arab, "Analytic Design Method Based on Homothetic Shape of Magnetic Cores for HighFrequency Transformers," in IEEE Transactions on Power Electronics, vol. 22, no. 5, pp. 2070-2080, Sept. 2007.

[11] (2019, Apr.) SciPy. [Online]. Available:https://scipy.org

[12] (2019, Apr.) Soft Ferrites and Accessories - Data Handbook 2013 [Online]. Available: https://www.ferroxcube.com [13] D. Meeker, Finite Element Method Magnetics, Version 4.2 (12Jan 20162006 Build).

[14] N. Ida and J. P. A. Bastos, Electromagnetics and Calculation of Fields, 2nd ed. NewYork, NY: Springer, 1997, p. 432. 
[15] D. Meeker, Finite element method magnetics, Version 4.2, Users Manual, October 2015. 